\title{
Exogenous Testosterone Increases Decoy Effect in Healthy Males
}

\author{
Jiajun Liao ${ }^{1,2}$, Yang Zhang ${ }^{3}$, Yingchun $L^{3}{ }^{3}$, Hong $L^{1 i^{1,2}}$, Samuele Zilioli4,5 and Yin $W u^{1,2 *}$ \\ 'Shenzhen Key Laboratory of Affective and Social Cognitive Science, Shenzhen University, Shenzhen, China, ${ }^{2}$ College \\ of Psychology and Sociology, Shenzhen University, Shenzhen, China, ${ }^{3}$ School of Science, Harbin Institute of Technology \\ (Shenzhen), Shenzhen, China, ${ }^{4}$ Department of Psychology, Wayne State University, Detroit, MI, United States, ${ }^{5}$ Department \\ of Family Medicine and Public Health Sciences, Wayne State University, Detroit, MI, United States
}

OPEN ACCESS

Edited by:

Bernd Weber,

Universität Bonn, Germany

Reviewed by:

Anthony John Porcelli,

Marquette University, United States

Ignacio Saez,

University of California, Berkeley,

United States

*Correspondence:

Yin Wu

yinwu0407@gmail.com

Specialty section:

This article was submitted to

Decision Neuroscience,

a section of the journal

Frontiers in Psychology

Received: 26 July 2018

Accepted: 23 October 2018

Published: 13 November 2018

Citation:

Liao J, Zhang Y, Li Y, Li H, Zilioli S and Wu Y (2018) Exogenous Testosterone Increases Decoy Effect in Healthy Males.

Front. Psychol. 9:2188. doi: 10.3389/fpsyg.2018.02188
There is increasing interest in the role played by testosterone in economic decisionmaking and social cognition. However, despite the growing body of findings in this field of research, no empirical study to date has tested whether testosterone modulates decision-making when an asymmetrically dominated decoy option is introduced in a choice set. Within a choice set that comprises two options, an asymmetrically dominated decoy option is a third option that, when introduced in the choice set, is much worse than one of the existing options, but comparable to the other existing option. Introduction of a decoy option leads to a preference toward the dominating option (i.e., decoy effect). Healthy male participants $(n=63)$ received a single-dose of $150 \mathrm{mg}$ testosterone gel in a double-blind, placebo-controlled, between-subjects design. At 180 min post-administration, participants took part in a decision-making task to elicit decoy effect. Results showed that participants in the testosterone group made less consistent choices and more target choices (i.e., decoy effect) than participants in the placebo group. These findings are interpreted in light of the dual-process theory and are in line with existing evidence suggesting that testosterone promotes more intuitive and automatic judgments in human decision-making.

Keywords: androgen, human male, decoy effect, preference, decision-making

\section{INTRODUCTION}

Testosterone is a sex steroid that, in addition to being involved in reproductive physiology and morphology, plays an important role in various psychological processes, including decision-making (Eisenegger et al., 2011). Higher testosterone levels have been associated with dominant behaviors, such as social aggression (Eisenegger et al., 2011) and risk-taking (Apicella et al., 2015), but also prosocial acts (van Honk et al., 2012). Recent evidence has reconciled these contrasting findings by invoking the moderating role played by contextual influences (Boksem et al., 2013; Dreher et al., 2016). Although the realization that testosterone influences decisions in a context-dependent fashion is relatively new, the broader idea that human choices are fine-tuned to the context in which options are presented has long been known (Kahneman, 2011). The "framing effect," the idea that individuals tend to be risk averse when options are presented in a gain frame and risk seeking when options are presented in a loss frame, is an example of how contextual influences affect decision making (Tversky and Kahneman, 1981).

Two recent studies highlight the flexible role of testosterone in decision making (Boksem et al., 2013; Dreher et al., 2016). In a modified Ultimatum Game, testosterone increased both punishment 
and reward of proposers, depending on proposers' offers. Using the Trust Game, Boksem et al. (2013) showed that testosterone modulated behavior depending on whether participants played the investor or the trustee role. Findings from these two studies support the hypothesis that testosterone flexibly influences decision-making in scenarios that involve multiple social actors. A gap in the literature is whether the observed contextdependent effects of testosterone on decision-making also extend to individual (vs. social) scenarios where contextual influences are related to how decision options are presented. This research question is particular relevant when we consider that many daily decisions happen in these individual scenarios.

One way to address this gap in the literature is to test whether and how testosterone modulates decision-making when an asymmetrically dominated decoy option is introduced in a choice set. Decoy effect refers to the phenomenon that adding a new option to an existing set of options highlights the superiority of one of the existing options, shifting individuals' preference toward that option (Huber et al., 1982). In other words, a decoy option is an additional option that is worse than one of the existing options, the dominating option, which, as a result, becomes the most attractive one. For example, as depicted in Figure 1A, a decision maker might be undecided about mobile battery A or mobile battery B. Mobile battery A has a larger capacity but is a bit more expensive, while mobile battery $\mathrm{B}$ has a lower capacity but is a bit cheaper. This impasse can be resolved by introducing option $\mathrm{C}$, which acts as a "decoy." Option $\mathrm{C}$ is better than option $B$ in terms of capacity, worse than option $B$ in terms of price, and, more importantly, worse than option A on both attributes (i.e., price and capacity). Thus, option $\mathrm{C}$ is the asymmetrically dominated decoy option, which boosts decision makers' preference toward option $\mathrm{A}$, the dominating option. Option B can become the dominating option if a different decoy option is introduced, as shown in Figure 1B. The decoy effect has been found in various domains of decision-making, including motor planning (Farmer et al., 2015), marketing strategy (Ariely, 2009), and behavioral nudge (Li et al., 2018). Here, we tested how testosterone modulated decision making in a decoy paradigm.

\section{MATERIALS AND METHODS}

\section{Participants}

Sixty-three healthy males (mean age $=21.3$ years, $S D=1.5$, age range $=19-26$ ) at Shenzhen University, China, were recruited through advertisement. Participants were screened over the phone and excluded if they were taking any psychotropic medication and had a history of psychiatric or neurological disorders. We tested only men because the dosing and pharmacokinetics of a single dose of Androgel used in the study have been established for men only (Eisenegger et al., 2013). Participants were instructed to abstain from alcohol, caffeine intake, and smoking for $24 \mathrm{~h}$ before the testing session. The study was conducted in accordance with the Declaration of Helsinki and was approved by the Shenzhen University Medical Research Ethics Committee. Written informed consent was obtained from all participants.

\section{Testosterone Administration}

The study used a double-blind, placebo-controlled, betweensubjects design. All sessions started at 13:00 and took about $4.5 \mathrm{~h}$ to complete. Participants in the testosterone condition received a single dose of testosterone gel, containing $150 \mathrm{mg}$ of testosterone [Androgel]. Participants in the placebo condition received a colorless hydroalcoholic gel. In both conditions, a male research assistant, who was blind to the purpose of the study, applied the gel to participants' shoulders and upper arms. The experimenter was unblinded after the data analysis was carried out. Due to the established time lag of $3 \mathrm{~h}$ for behavioral effects following testosterone gel application in healthy males (Eisenegger et al., 2013; Carré et al., 2015), the choice task commenced $3 \mathrm{~h}$ post-dosing. Participants also completed two additional decision-making tasks, which are not reported here.

\section{The Decision-Making Task}

Participants completed a decoy decision-making task, which was adapted from Farmer et al. (2017). The task was programmed using E-Prime (version 2.0; Psychology Software Tools, Inc., PA, United States). Participants were presented with 10 pairs of products. Products in each pair were different on two attributes (e.g., Figures 1A,B). Each pair was presented twice, once with a decoy that targeted one product (Figure 1A) and once with a decoy that targeted the other product (Figure 1B); in addition, on six catch trials, one of the three products was clearly superior to the two other products (see Figure 1C). All three products (i.e., target, competitor, and decoy) were presented on each trial along with text describing their attributes. Screen locations were randomized in each trial. Using the computer keyboard, participants had to indicate their preferred product. No time constraint was imposed on choice selection. Trial order was randomized, with the only constraint that all pairs of products (and decoy) had to be presented once before being presented a second time (with a different decoy).

\section{Data Analysis}

Participants' choices were assigned to one of four categories (see also, Farmer et al., 2017). Consistent choices were those in which participants chose the same option when presented with the same pair of products, regardless of the decoy. Target choices were those in which participants chose the option targeted by the decoy (i.e., dominating option) (e.g., mobile battery A in Figure 1A). Non-target choices were those in which participants chose the option that was not targeted by the decoy in both presentations (e.g., mobile battery B in Figure 1A). Decoy choices were cases in which the participant chose the decoy on one or both presentations of a given product pair. Proportions in each category were calculated and compared as a function of the experimental condition (i.e., testosterone vs. placebo) using independent-samples $t$-tests.

\section{RESULTS}

We excluded five participants who exhibited excessive error rate $(>50 \%)$ in catch trials (i.e., failing to choose 

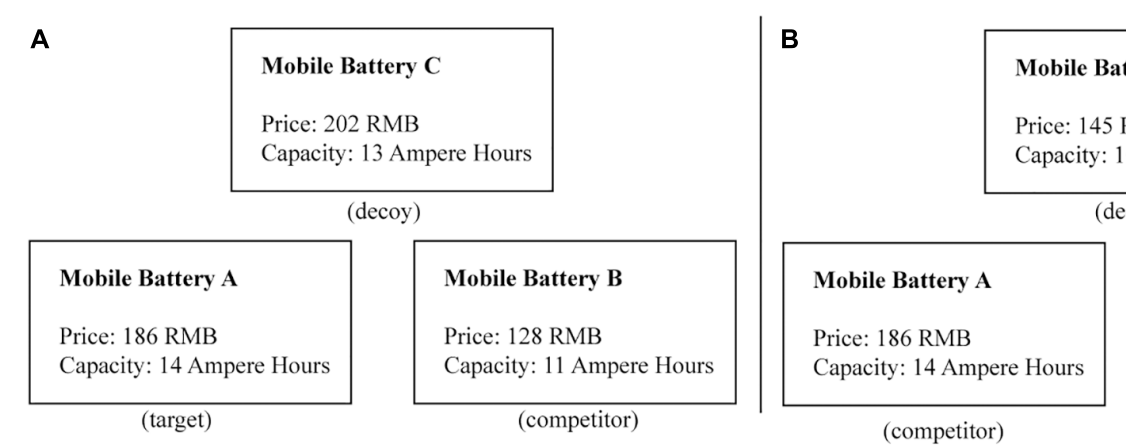

c

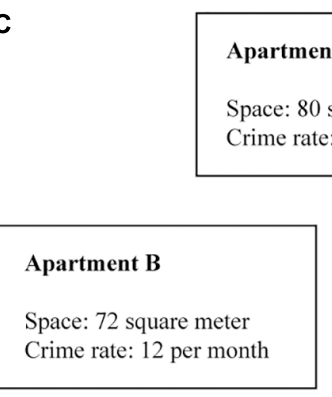

(competitor)

\section{partment $A$}

ace: 80 square meter

me rate: 14 per month obile Battery C

rice: $145 \mathrm{RMB}$

pacity: 10 Ampere Hours

(decoy)

\begin{tabular}{|l|}
\hline Mobile Battery B \\
Price: 128 RMB \\
Capacity: 11 Ampere Hours \\
\hline (target)
\end{tabular}

FIGURE 1 | (A) The product on the lower left is the target, the product on the lower right is the competitor, and the product on the top is the decoy. (B) The product on the lower right is the target, the product on the lower left is the competitor, and the product on the top is the decoy. (C) A catch trial example, the product on the lower right is clearly superior to the two other products.

the product that was clearly superior to the other two products). As shown in Figure 2, participants in the testosterone condition $(M=61.03 \%, S D=17.80 \%)$ made less consistent choices than participants in the placebo condition $(M=72.41 \%, S D=15.27 \%)$, $t(56)=-2.613, p=0.012, d=0.688,95 \%$ confidence interval $(\mathrm{CI})=[-0.201,-0.027]$. Testosterone administration also increased the proportion of target choices

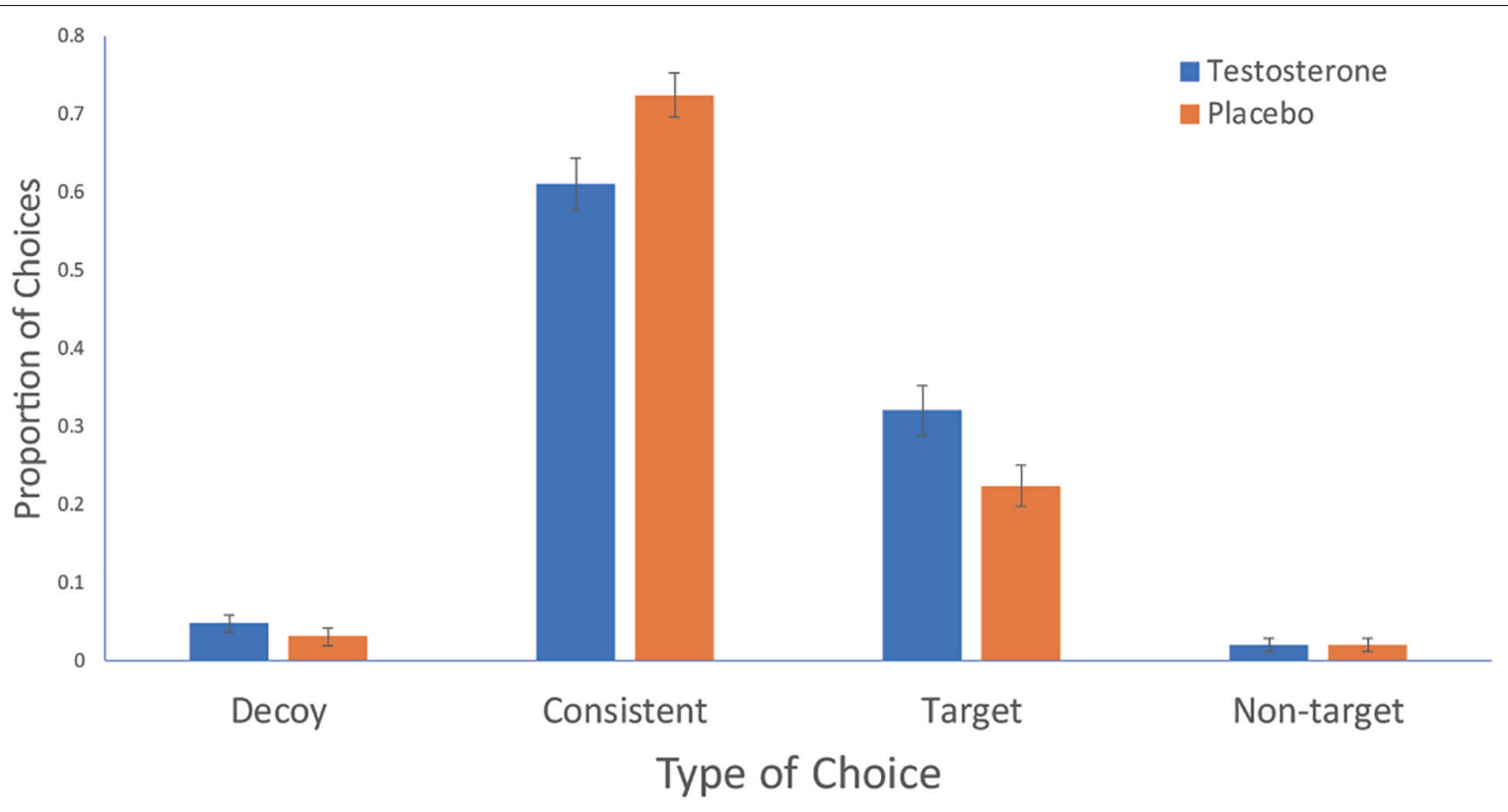

FIGURE 2 | Mean proportion of choices among four possible types of choices. Error bars represent standard errors of the mean. 
$[t(56)=2.308, p=0.025, d=0.602,95 \% \mathrm{CI}=(0.013$, $0.180)$; testosterone $(M=32.07 \%, S D=17.40 \%)$ vs. placebo $(M=22.41 \%, S D=14.31 \%)]$. Participants in the testosterone and placebo conditions did not differ in terms of non-target choices $(M=2.10 \%, S D=4.91 \%$ vs. $M=2.10 \%, S D=4.12 \%$, $p>0.1)$ and decoy choices $(M=4.83 \%, S D=6.34 \%$ vs. $M=3.10 \%, S D=6.04 \%, p>0.1)$. The same analyses were re-run including the five participants who exhibited excessive error rate, and the same pattern of results emerged, with participants in the testosterone condition making more target choices than participants in the placebo condition $[t(61)=2.643$, $p=0.010, d=0.665,95 \% \mathrm{CI}=(0.025,0.182)$, testosterone $(M=31.61 \%, S D=16.95 \%)$ vs. placebo $(M=21.25 \%$, $S D=14.09 \%)]$.

In a set of ancillary analyses, differences in reaction time (RT) were compared as a function of the experimental condition. For these analyses, we excluded trials in which RTs exceeded by three standard deviations participants' mean RT. Following this procedure, $1.23 \%$ of the total data points were excluded. RT values were log-transformed in order to achieve normality. Participants in the testosterone condition $\left(M=11,501 \mathrm{~ms}^{1}, S D=5,007\right)$ and placebo condition $(M=9,800 \mathrm{~ms}, S D=3,859)$ did not differ on the RT, $t(56)=1.342$, $p=0.185$.

\section{DISCUSSION}

Using a decoy paradigm, we found that testosterone administration reduced consistency in decision-making and increased the selection of dominating options (i.e., decoy effect). These findings are in agreement with previous work on the context-dependent nature of testosterone effects on social decision-making (Boksem et al., 2013; Dreher et al., 2016). Notably, the present study, which employed a decoy paradigm that did not involve other individuals, extends the existing literature on the flexible effects of testosterone on decision-making in social scenarios to individual scenarios. The present finding suggests that testosterone promotes behavioral flexibility by fine-tuning behaviors in response to the environment, a hypothesis recently corroborated in a correlational study testing the association between endogenous levels of testosterone and behavioral flexibility using a stimulusoutcome reversal learning paradigm (Diekhof and Kraft, 2017).

${ }^{1}$ Note that the descriptive statistics of RTs are not transformed.

\section{REFERENCES}

Apicella, C. L., Carré, J. M., and Dreber, A. (2015). Testosterone and economic risk taking: a review. Adapt. Hum. Behav. Physiol. 1, 358-385. doi: 10.1007/s40750014-0020-2

Ariely, D. (2009). Predictably Irrational: The Hidden Forces That Shape Our Decisions. New York, NY: HarperCollins.

Boksem, M. A. S., Mehta, P. H., Van den Bergh, B., van Son, V., Trautmann, S. T., Roelofs, K., et al. (2013). Testosterone inhibits trust but promotes reciprocity. Psychol. Sci. 24, 2306-2314. doi: 10.1177/0956797613495063
Our findings can be read in light of the dual-process theory, according to which two thought processes characterize human decision-making (Kahneman, 2011). The first process consists of rapid, automatic, emotional, and intuitive processes (i.e., System 1 ), while the second process is characterized by slow, effortful, and deliberate processes (i.e., System 2). Recent work on the decoy effect showed that individuals with a greater proclivity to intuitive reasoning (i.e., System 1) were more likely to be influenced by a decoy. Similarly, Pocheptsova et al. (2009) found that cognitive load, which impairs deliberation and increased reliance on intuitive processing, exacerbated the decoy effect. The present finding is also consistent with previous research showing that testosterone shifts the balance between System 1 and System 2, making individuals more reliant on intuitive decisionmaking. Another study showed that testosterone administration amplified emotional influences (i.e., anticipatory regret) on decision making and increased affective sensitivity to decision outcome (Wu et al., 2018). Taken together, these findings suggest that testosterone promotes intuitive and automatic judgment in human decision-making.

\section{AUTHOR CONTRIBUTIONS}

JL, HL, and YW developed the concepts for the study. JL and YW collected the data. JL and YW analyzed the data. All authors contributed to the manuscript and approved the final version of the manuscript for submission.

\section{FUNDING}

This work was supported by the National Natural Science Foundation of China (31872784, 31600923, and 31671150), Shenzhen University Teaching Fund (JG2018084), and Shenzhen University Social and Humanity Science Research Fund (17QNFC44) to YW, and the Shenzhen Peacock Plan (KQTD2015033016104926) to HL. The funding sources had no further role in the study design, data collection, analysis, interpretation, and decision to submit this manuscript for publication.

\section{ACKNOWLEDGMENTS}

The authors are grateful to Dr. Jinting Liu, Mr. Yizhi Cheng, and Mr. Zhiwei Wu for their help with the study.

Carré, J. M., Ortiz, T. L., Labine, B., Moreau, B. J. P., Viding, E., Neumann, C. S., et al. (2015). Digit ratio (2D:4D) and psychopathic traits moderate the effect of exogenous testosterone on socio-cognitive processes in men. Psychoneuroendocrinology 62, 319-326. doi: 10.1016/j.psyneuen.2015.08.023

Diekhof, E. K., and Kraft, S. (2017). The association between endogenous testosterone level and behavioral flexibility in young men - evidence from stimulus-outcome reversal learning. Horm. Behav. 89, 193-200. doi: 10.1016/ j.yhbeh.2017.02.006

Dreher, J., Dunne, S., Pazderska, A., Frodl, T., Nolan, J. J., and O’Doherty, J. P. (2016). Testosterone causes both prosocial and antisocial status-enhancing 
behaviors in human males. Proc. Natl. Acad. Sci. U.S.A. 113, 11633-11638. doi: $10.1073 /$ pnas. 1608085113

Eisenegger, C., Haushofer, J., and Fehr, E. (2011). The role of testosterone in social interaction. Trends Cogn. Sci. 15, 263-271. doi: 10.1016/j.tics.2011.04.008

Eisenegger, C., von Eckardstein, A., Fehr, E., and von Eckardstein, S. (2013). Pharmacokinetics of testosterone and estradiol gel preparations in healthy young men. Psychoneuroendocrinology 38, 171-178. doi: 10.1016/j.psyneuen. 2012.05.018

Farmer, G. D., Baron-Cohen, S., and Skylark, W. J. (2017). People with autism spectrum conditions make more consistent decisions. Psychol. Sci. 28, 1067-1076. doi: 10.1177/0956797617694867

Farmer, G. D., El-Deredy, W., Howes, A., and Warren, P. A. (2015). The attraction effect in motor planning decisions. Judgm. Decis. Mak. 10, 503-510.

Huber, J., Payne, J. W., and Puto, C. (1982). Adding asymmetrically dominated alternatives: violations of regularity and the similarity hypothesis. J. Consum. Res. 9, 90-98. doi: 10.1086/208899

Kahneman, D. (2011). Thinking, Fast and Slow. New York, NY: Penguin.

Li, M., Sun, Y., and Chen, H. (2018). The decoy effect as a nudge: boosting hand hygiene with a worse option. Psychol. Sci. 095679761876137. (in press). doi: 10.1177/0956797618761374

Pocheptsova, A., Amir, O., Dhar, R., and Baumeister, R. F. (2009). Deciding without resources: resource depletion and choice in context. J. Mark. Res. 46, 344-355. doi: $10.1509 / j m k r .46 .3 .344$
Tversky, A., and Kahneman, D. (1981). The framing of decisions and the psychology of choice. Science 211, 453-458. doi: 10.1126/science.745 5683

van Honk, J., Montoya, E. R., Bos, P. A., van Vugt, M., and Terburg, D. (2012). New evidence on testosterone and cooperation. Nature 485, E4-E5. doi: 10.1038/nature11136

Wu, Y., Clark, L., Zilioli, S., Eisenegger, C., Gillan, C. M., Deng, H., et al. (2018). Single dose testosterone administration modulates emotional reactivity and counterfactual choice in healthy males. Psychoneuroendocrinology 90, 127-133. doi: 10.1016/j.psyneuen.2018. 02.018

Conflict of Interest Statement: The authors declare that the research was conducted in the absence of any commercial or financial relationships that could be construed as a potential conflict of interest.

Copyright (c) 2018 Liao, Zhang, Li, Li, Zilioli and Wu. This is an open-access article distributed under the terms of the Creative Commons Attribution License (CC BY). The use, distribution or reproduction in other forums is permitted, provided the original author(s) and the copyright owner(s) are credited and that the original publication in this journal is cited, in accordance with accepted academic practice. No use, distribution or reproduction is permitted which does not comply with these terms. 